\title{
Improved molecular classification of serrated lesions of the colon by immunohistochemical detection of BRAF V600E
}

\author{
Ildiko Mesteri ${ }^{1}$, Günther Bayer ${ }^{1}$, Jochen Meyer $^{2,3}$, David Capper ${ }^{2,3}$, \\ Sebastian F Schoppmann ${ }^{4}$, Andreas von Deimling ${ }^{2,3}$ and Peter Birner ${ }^{1,2}$ \\ ${ }^{1}$ Clinical Institute of Pathology, Medical University of Vienna, Vienna, Austria; ${ }^{2}$ Department of \\ Neuropathology, Institute of Pathology, Ruprecht-Karls-University Heidelberg, Heidelberg, Germany; \\ ${ }^{3}$ Clinical Cooperation Unit Neuropathology, German Cancer Research Centre, Heidelberg, Germany and \\ ${ }^{4}$ Department of Surgery, Medical University of Vienna, Vienna, Austria
}

\begin{abstract}
BRAF V600E mutation in serrated lesions of the colon has been implicated as an important mutation and as a specific marker for the serrated carcinogenic pathway. Recent findings point to microvesicular hyperplastic polyps that have similar histologic and molecular features to sessile serrated adenomas/polyps, as potential colorectal carcinoma precursors. The aim of this study was to evaluate BRAF V600E mutation status by immunohistochemistry in serrated lesions of the colon with regard to histomorphology. We investigated 194 serrated lesions of the colon, comprising 42 sessile serrated adenomas/polyps, 16 traditional serrated adenomas, 136 hyperplastic polyps and 20 tubular/tubulovillous adenomas (conventional adenomas) with the novel BRAF V600E mutation-specific antibody VE1. In addition, BRAF exon 15 and KRAS exon 2 status was investigated by capillary sequencing in selected cases. All sessile serrated adenomas/polyps (42/42, 100\%), 15/ $16(94 \%)$ traditional serrated adenomas and 84/136 (62\%) hyperplastic polyps were VE1 + . None of the VE1 serrated lesions showed BRAF V600E mutation. Forty out of $42(95 \%)$ sessile serrated adenomas/polyps displayed areas with microvesicular hyperplastic polyp-like features. In microvesicular hyperplastic polyps, VE1 positivity was significantly associated with nuclear atypia $(P=0.003)$; however, nuclear atypia was also present in VE1 - cases. Immunostaining with VE1 allows not only the detection of BRAF V600E mutation but also the correlation with histomorphology on a cellular level in serrated lesions. VE1 enables a subclassification of microvesicular hyperplastic polyps according to the mutation status. This improved classification of serrated lesions including immunohistochemical evaluation of BRAF V600E mutation may be the key to identify lesions with higher potential to progression into sessile serrated adenoma/polyp, and further to BRAF V600Emutated colorectal cancer.
\end{abstract}

Modern Pathology (2014) 27, 135-144; doi:10.1038/modpathol.2013.126; published online 26 July 2013

Keywords: BRAF; colon; immunohistochemistry; morphology; serrated polyp

Colorectal cancer is one of the leading causes of cancer death worldwide. Although increased use of screening and surveillance, as well as detection and the removal of conventional adenomas, has led to a reduction in the incidence and mortality of this disease, this effect is mainly limited to distal colorectal cancer. ${ }^{1-5}$ However, difficulties remain in the prevention of proximal colorectal cancer.

Correspondence: Professor P Birner, MD, MSc, Clinical Institute of Pathology, Medical University of Vienna, Währinger Gürtel 18-20, 1090 Vienna, Austria.

E-mail: peter.birner@meduniwien.ac.at

Received 8 November 2012; accepted 3 June 2013; published online 26 July 2013
A recent study suggested that colorectal cancers of the proximal colon develop in a significantly shorter period of time than those in the distal colon, after a patient has had a negative colonoscopy. ${ }^{2,3}$ Thus, there is a need for improved and targeted identification of neoplastic precursor lesions in the proximal colon.

Colorectal cancer arises from several different types of precursor lesions. In the conventional pathway, carcinomas develop from adenomas via biallelic APC (adenomatous polyposis coli) mutations $^{6}$ and are reinforced by KRAS mutation.

In the other major pathway, known as the serrated carcinogenic pathway, tumors develop from serrated precursor lesions via BRAF mutations. ${ }^{7-9}$ Serrated 
precursor lesions consist of sessile serrated adenomas/polyps (either with or without cytologic dysplasia) and traditional serrated adenomas. ${ }^{9-12}$ Serrated lesions constitute the main precursor lesion in at least one-third of all colorectal cancers. ${ }^{13,14}$ Progression results in microsatellite-unstable colorectal cancer and possibly also CpG island-methylated microsatellitestable carcinomas. ${ }^{3,9-12}$ Although consensus exists that sessile serrated adenomas/polyps and traditional serrated adenomas are colorectal cancer precursor lesions, ${ }^{13}$ hyperplastic polyps were considered for decades to have no malignant potential.

This has been questioned in the past years and different subsets of hyperplastic polyps with distinct molecular profiles and potential to progress to colorectal cancer have been identified. ${ }^{15}$ These findings implicate that BRAF V600E-mutated colorectal cancers have their origin in hyperplastic polyps classified morphologically as microvesicular hyperplastic polyps. ${ }^{9,15,16}$ Should this be correct, a refined classification of microvesicular hyperplastic polyps with regard to $B R A F$ V600E mutation might lead to an improved assessment of colorectal cancer risk and guide patient management.

In contrast to conventional adenomas where cytological changes have an important role in identifying dysplasia, diagnosis of the various subclasses of serrated lesions is mainly based on the architectural criteria. ${ }^{17,18}$ Owing to sampling issues, poor specimen orientation and a significant interobserver variation among pathologists, ${ }^{19-22}$ the differential diagnosis of microvesicular hyperplastic polyps vs sessile serrated adenomas/polyps can be very challenging or even impossible. Especially, if we consider that for the differential diagnosis of microvesicular hyperplastic polyp vs sessile serrated adenoma/polyp, the presence of the appropriate morphologic criteria in just one crypt is considered as sufficient. ${ }^{13}$

It has been suggested that $B R A F$ mutation is one of the important player in two of the five molecular subtypes of colorectal cancer according to Jass. ${ }^{23}$ Currently, there are no established morphologic criteria that would identify oncogenic BRAF V600E mutation in serrated lesions. Until recently, assessment of BRAF V600E mutation status was not feasible in routine pathology, as sequencing of all serrated lesions is not suitable for everyday diagnostic pathology. In addition, direct sequencing might provide false-negative results owing to dilution of the diagnostic mutation through a high number of normal mucosa cells. In this regard, even microdissection cannot give proper evidence of the distribution of cells with BRAF V600E mutation within serrated lesions.

The development of a novel, mutation-specific antibody has enabled us to investigate the presence of $V 600 E$-mutated $B R A F$ protein in formalin-fixed, paraffin-embedded sections. ${ }^{24-26}$ The aim of this study was to evaluate BRAF V600E protein expression in serrated lesions of the colon in correlation with morphology. The expression of BRAF V600E protein in microvesicular hyperplastic polyps (with and without nuclear atypia), sessile serrated adenomas/polyps (with and without cytologic dysplasia) and traditional serrated adenomas should aid in subclassifying these lesions also according to their BRAF V600E mutation status in addition to histomorphologic classification. In routine pathology, this would identify serrated lesions carrying the oncogenic BRAF V600E mutation, which might have an increased risk of progression to colorectal cancer. The implementation of a new classification based on distinct molecular phenotype could offer patients an individualized cancer prevention and therapy option.

\section{Materials and methods}

\section{Tissue Samples}

Institutional review board approval was obtained for this study.

Formalin-fixed, paraffin-embedded samples of serrated, adenomatous and hyperplastic colon lesions were identified in the archives of the Clinical Institute for Pathology of the Medical University of Vienna (Vienna, Austria). Samples that were improperly oriented or did not contain sufficient amounts of tissue for further analysis were excluded from the study. Before the immunohistochemical evaluation with VE1 antibody, all tissue samples were reviewed and histologically subclassified on hematoxylin- and eosin-stained slides according to the current WHO scheme. ${ }^{27}$

In brief, classification was based on the following criteria:

Goblet-cell-rich hyperplastic polyp: comprises pure goblet cells, straight crypts with proliferative activity confined to the lower crypt, showing minimal serration.

Microvesicular hyperplastic polyp: comprises epithelial cells with hypermucinous microvesicular cytoplasm only or mixed with goblet cells with maintained maturation towards the surface. Crypts are straight, dilatation/serration is confined in the more luminal aspects, crypt bases are narrow with symmetrical proliferative activity and interspersed neuroendocrine cells.

Sessile serrated adenoma/polyp: The criteria for the diagnosis, as suggested by an expert panel, ${ }^{13}$ was the minimum of one crypt that clearly showed dilatation and/or serration extending to the crypt base, with or without branching (L-, inverted T- or anchor-shape) of the crypts and abnormal proliferation (proliferative zone located on the side, frequently asymmetrical) showing goblet cells or gastric foveolar cells in the crypt basis. In addition, absence or presence of cytologic dysplasia were determined. 
a
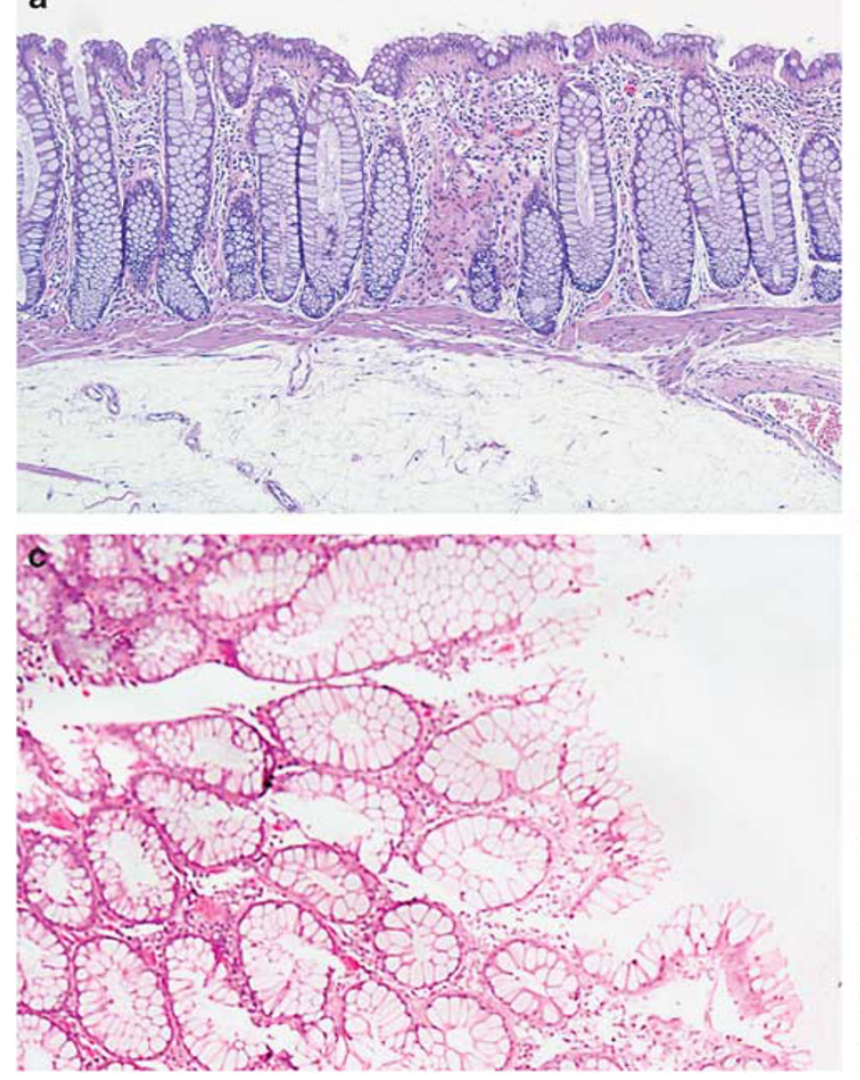

b
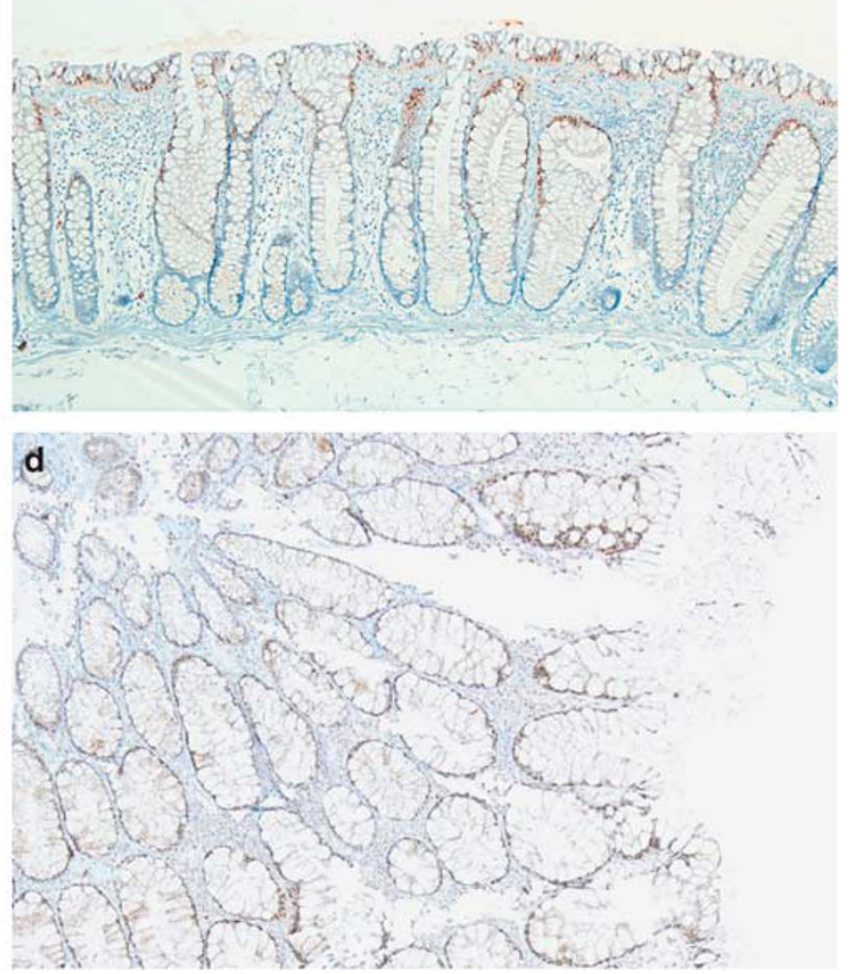

Figure 1 Normal colonic mucosa: (a) hematoxylin and eosin (H\&E), $\times 100$; (b) Immunostain VE1, $\times$ 100. Goblet-cell-rich hyperplastic polyp: (c) H\&E, $\times 200$; (d) Immunostain VE1, $\times 200$.

Traditional serrated adenoma: shows villiform growth pattern with serration due to infolding, budding and papillary tufting, composed of tall columnar cells with pencillated nucleus and hypereosinophilic cytoplasm with interspersed goblet cells. In addition, the grade of cytologic dysplasia was determined.

In total, 194 consecutive serrated lesions of the colon were included in our study, these included: 42 sessile serrated adenomas/polyps (38 without cytologic dysplasia and 4 with cytologic dysplasia), 16 traditional serrated adenomas with low-grade dysplasia, 119 microvesicular hyperplastic polyps and 17 goblet-cell rich hyperplastic polyp. Additionally for comparison, 20 tubular/tubulovillous (conventional adenomas) with low-grade dysplasia mainly from the right colon were also included. Mucin-poor-type hyperplastic polyps were not found in our series.

Microvesicular hyperplastic polyps were evaluated for the degree of nuclear atypia according to the schema of Torlakovic et al. ${ }^{17}$ In addition, sessile serrated adenomas/polyps were reviewed for the presence of areas revealing a morphology of microvesicular hyperplastic polyps.

The classification was carried out by three independent pathologists (IM, GB and PB), with consecutive consensus for each case.
Immunohistochemistry. A Benchmark ultraimmunostainer was used for immunohistochemistry (Ventana, Tucson, AZ, USA).

Expression of V600E-mutated BRAF was evaluated in 3- $\mu \mathrm{m}$-thick histological slides using the monoclonal BRAF V600E mutation-specific antibody VE1 (provided by AvD, the antibody is commercially available from Spring Bioscience, Pleasanton, CA, USA). Production and validation of this antibody has been published in detail previously. ${ }^{28} \mathrm{~A}$ specimen was considered positive for BRAF V600E if distinct cytoplasmic staining reaction with the VE1 antibody was present (samples of immunostaining are given in Figures 1-4). ${ }^{24,26}$ Nonspecific nuclear staining without cytoplasmic signal was detected in the surface epithelium of adjacent normal colon mucosa (Figure 1). This nuclear staining pattern has also been previously reported in brain metastases, but in that study this type of staining showed no correlation with BRAF mutation status. ${ }^{26}$

To exclude the possibility that nuclear staining pattern with VE1 in normal mucosa corresponded to the presence of BRAF protein, cases with sufficient material available from VE1 - hyperplastic polyps, which showed nonspecific nuclear staining, were immunostained with a pan-BRAF-antibody detecting total (mutated and wild-type) BRAF (pBR1, dilution 1:4). ${ }^{28}$ In these cases, only cytoplasmic, but 
Table 1 Results of VE1 immunohistochemistry in all $(n=214)$ and $K R A S$ sequencing in selected $(n=41)$ samples

\begin{tabular}{|c|c|c|c|}
\hline Diagnosis & Localization & $B R A F$ VE1 + & KRAS \\
\hline Sessile serrated adenoma/polyp & $\begin{array}{l}\text { All localizations }(n=42) \\
\text { Right colon }(n=32) \\
\text { C. transversum }(n=4) \\
\text { Left colon }(n=6)\end{array}$ & $\begin{array}{c}42 / 42(100 \%) \\
32 / 32(100 \%) \\
4 / 4(100 \%) \\
6 / 6(100 \%)\end{array}$ & $0 / 4(0 \%)$ \\
\hline Traditional serrated adenoma & $\begin{array}{l}\text { All localizations }(n=16) \\
\text { Right colon }(n=2) \\
\text { C. transversum }(n=0) \\
\text { Left colon }(n=14)\end{array}$ & $\begin{array}{c}15 / 16(94 \%) \\
2 / 2(100 \%) \\
- \\
13 / 14(93 \%)\end{array}$ & $0 / 2(0 \%)$ \\
\hline $\begin{array}{l}\text { Hyperplastic polyp } \\
\text { Microvesicular hyperplastic polyp }\end{array}$ & $\begin{array}{l}\text { All localizations }(n=136) \\
\text { All localizations }(n=119) \\
\text { Right colon }(n=48) \\
\text { C. transversum }(n=18) \\
\text { Left colon }(n=53)\end{array}$ & $\begin{array}{c}84 / 136(62 \%) \\
84 / 119(71 \%) \\
39 / 48(81 \%) \\
12 / 18(67 \%) \\
33 / 53(62 \%)\end{array}$ & $\begin{array}{l}2 / 31(6 \%) \\
2 / 20(10 \%)\end{array}$ \\
\hline Goblet-cell-rich hyperplastic polyp & $\begin{array}{l}\text { All localizations }(n=17) \\
\text { Right colon }(n=17) \\
\text { C. transversum }(n=0) \\
\text { Left colon }(n=0)\end{array}$ & $\begin{array}{l}0 / 17(0 \%) \\
0 / 17(0 \%) \\
-\end{array}$ & $0 / 11(0 \%)$ \\
\hline $\begin{array}{l}\text { Mucin-poor hyperplastic polyp } \\
\text { Tubulary/tubulovillous adenoma }\end{array}$ & $\begin{array}{l}\text { All localizations }(n=0) \\
\text { All localizations }(n=20) \\
\text { Right colon }(n=12) \\
\text { C. transversum }(n=2) \\
\text { Left colon }(n=6)\end{array}$ & $\begin{array}{c}- \\
0 / 20(0 \%) \\
0 / 12(0 \%) \\
0 / 2(0 \%) \\
0 / 6(0 \%)\end{array}$ & $1 / 4 \overline{(25} \%)$ \\
\hline
\end{tabular}

no nuclear, staining was evident, indicating a lack of BRAF in the nucleus. The nuclear staining reaction did not interfere with the determination of BRAF status, as mutated BRAF protein is evident by strong cytoplasmic staining.

Sequence analysis of KRAS and BRAF. BRAF exon 15 and $K R A S$ exon 2 (56 of 115) were investigated by capillary sequencing after macrodissection of specimens. Preparation of DNA and sequencing of $B R A F$ exon 15 was performed as described previously. ${ }^{24}$ Briefly, a fragment spanning exon 2 of KRAS (sense: $5^{\prime}$-GCCTGCTGAAAATGACTGAA-3'; antisense: $5^{\prime}$-AGAATGGTCCTGCACCAGTAA-3') was amplified using $20 \mathrm{ng}$ each of the respective forward and reverse primer. Primer design was based on accession number NG_007524.1. For PCR, 50 ng of DNA and HotStar $2 \times$ PCR Master Mix (Qiagen, Hilden, Germany) were used. PCR was performed in a total volume of $25 \mu \mathrm{l}$, and included initial denaturation at $95{ }^{\circ} \mathrm{C}$ for $180 \mathrm{~s}$, followed by 37 cycles with denaturation at $95^{\circ} \mathrm{C}$ for $30 \mathrm{~s}$, annealing at $56{ }^{\circ} \mathrm{C}$ for $25 \mathrm{~s}$ and extension at $72{ }^{\circ} \mathrm{C}$ for $40 \mathrm{~s}$. Two microliters of the amplification product were submitted to bidirectional sequencing using the BigDye Terminator v.3.1 Sequencing Kit (Applied Biosystems, Foster City, CA, USA). Sequences were determined using an ABI 3500 Genetic Analyzer (Applied Biosystems) and the Sequence-Pilot v. 3.4 (JSI-Medisys, Kippenheim, Germany) software.

Statistics. For statistical analysis, $\chi^{2}$ tests were used as appropriate; SPSS 20.0 (IBM, Armonk, NY, USA) was used for this purpose. All $P$-values given are results from two-tailed test, a $P$-value $\leq 0.05$ was considered as significant.

\section{Results}

Table 1 provides details on polyp type, anatomic localization and results of VE1 immunohistochemistry, as well as KRAS sequencing in selected samples.

All patients were Caucasian, of them 103 (48\%) female and 111 (52\%) male, median age 63 years (range 27-92 years) and mean age (years) $62 \pm 11$ (s.d.).

\section{Immunohistochemistry}

In VE1 + cases, a strong granular cytoplasmic staining of the epithelial cells was present. In sessile serrated adenoma/polyp without cytologic dysplasia, a more intense staining was detected in the basal half of the crypts compared with the superficial half. The intensity of the staining decreased continuously from the base of the crypts towards the surface epithelium in sessile serrated adenoma/polyp, which was stronger than that in microvesicular hyperplastic polyps. In traditional serrated adenoma and in sessile serrated adenoma/polyp with cytologic dysplasia, a diffuse strong intense staining was present with consistent strong expression continuously from the base of the crypts up to the surface epithelium. In all VE1 + lesions (microvesicular hyperplastic polyps, traditional serrated adenomas, sessile serrated adenomas/polyps), an abrupt transition could be observed between cells with and without BRAF V600E mutation. 
Table 2 VE1 positivity according to localization of lesions

\begin{tabular}{|c|c|c|}
\hline Localization & Diagnosis & $B R A F$ VE1 + \\
\hline \multirow[t]{6}{*}{ Right colon } & All & \\
\hline & Sessile serrated adenoma/polyp & $32 / 32(100 \%)$ \\
\hline & Traditional serrated adenoma & $2 / 2(100 \%)$ \\
\hline & $\begin{array}{l}\text { Microvesicular hyperplastic } \\
\text { polyp }\end{array}$ & $39 / 48(81 \%)$ \\
\hline & $\begin{array}{l}\text { Goblet-cell-rich hyperplastic } \\
\text { polyp }\end{array}$ & $0 / 17(0 \%)$ \\
\hline & $\begin{array}{l}\text { Tubulary/tubulovillous } \\
\text { adenoma }\end{array}$ & $0 / 12(0 \%)$ \\
\hline \multirow{6}{*}{$\begin{array}{l}\text { Colon } \\
\text { transversum }\end{array}$} & All & \\
\hline & Sessile serrated adenoma/polyp & $4 / 4(100 \%)$ \\
\hline & Traditional serrated adenoma & - \\
\hline & $\begin{array}{l}\text { Microvesicular hyperplastic } \\
\text { polyp }\end{array}$ & $12 / 18(67 \%)$ \\
\hline & $\begin{array}{l}\text { Goblet-cell-rich hyperplastic } \\
\text { polyp }\end{array}$ & - \\
\hline & $\begin{array}{l}\text { Tubulary/tubulovillous } \\
\text { adenoma }\end{array}$ & $0 / 2(0 \%)$ \\
\hline \multirow[t]{6}{*}{ Left colon } & All & \\
\hline & Sessile serrated adenoma/polyp & 6/6 $(100 \%)$ \\
\hline & Traditional serrated adenoma & $13 / 14(93 \%)$ \\
\hline & $\begin{array}{l}\text { Microvesicular hyperplastic } \\
\text { polyp }\end{array}$ & $33 / 53(62 \%)$ \\
\hline & $\begin{array}{l}\text { Goblet-cell-rich hyperplastic } \\
\text { polyp }\end{array}$ & - \\
\hline & $\begin{array}{l}\text { Tubulary/tubulovillous } \\
\text { adenoma }\end{array}$ & $0 / 6(0 \%)$ \\
\hline
\end{tabular}

Table 3 Sessile serrated adenoma/polyp showing microvesicular hyperplastic polyp-like features

\begin{tabular}{lcc}
\hline $\begin{array}{l}\text { Sessile serrated adenoma/polyp } \\
(\mathrm{n}=42)\end{array}$ & $\begin{array}{c}\text { Number of } \\
\text { cases }\end{array}$ & $\begin{array}{c}\% \text { VE1 }+ \\
\text { cases }\end{array}$ \\
\hline $\begin{array}{l}\text { Microvesicular hyperplastic } \\
\text { polyp-like features }\end{array}$ & $40 / 42$ & 100 \\
$\begin{array}{l}\text { Sessile serrated adenoma/polyp- } \\
\text { like areas }\end{array}$ & $42 / 42$ & 100 \\
\hline
\end{tabular}

One hundred and forty-one of 194 serrated lesions $(73 \%)$ showed specific cytoplasmic staining for VE1: $100 \%$ sessile serrated adenomas/polyps, $94 \%$ of traditional serrated adenomas and $62 \%$ of hyperplastic polyps. Of the 136 hyperplastic polyps, all 17 goblet-cell-rich hyperplastic polyps were negative for VE1 and $84(71 \%)$ of the 119 microvesicular hyperplastic polyps were positive for VE1. No significant difference of VE1 expression was observed between microvesicular hyperplastic polyps of the left colon compared with the right colon ( $P>0.05, \chi^{2}$ test; Table 2$)$.

All conventional adenomas were negative for VE1. Forty of 42 sessile serrated adenomas/polyps (95\%) showed areas with histological features of microvesicular hyperplastic polyps and a continuous positive VE1 expression, displaying significant correlation of microvesicular hyperplastic polyp-like features with BRAF V600E mutation (Table 3).
Table 4 Correlation of nuclear atypia in hyperplastic polyps with VE1 status

\begin{tabular}{lrr}
\hline Diagnosis & $\begin{array}{c}\text { No nuclear } \\
\text { atypia }\end{array}$ & $\begin{array}{c}\text { Nuclear } \\
\text { atypia }\end{array}$ \\
\hline $\begin{array}{l}\text { Microvesicular hyperplastic polyp } \\
(n=119)\end{array}$ & $44 / 119(37 \%)$ & $75 / 119(63 \%)$ \\
VE1 $-(n=35)$ & $20 / 35(57 \%)$ & $15 / 35(43 \%)$ \\
VE1 $+(n=84)$ & $24 / 84(29 \%)$ & $60 / 84(71 \%)$ \\
Goblet-cell-rich hyperplastic & $17 / 17(100 \%)$ & $0(0 \%)$ \\
$\begin{array}{l}\text { polyp }(n=17) \\
\text { VE1 }-(n=17)\end{array}$ & $17 / 17(100 \%)$ & $0(0 \%)$ \\
VE1 $+(n=0)$ & $0(0 \%)$ & $0(0 \%)$ \\
\hline
\end{tabular}

Only minimal nuclear atypia (Grade $1+$ according to Torlakovic et $a{ }^{17}$ ) and mild nuclear stratification was present in 75 hyperplastic polyps (55\%; 75 microvesicular hyperplastic polyps, 0 goblet-cell rich hyperplastic polyps). VE1 expression was seen in 84 $(71 \%)$ of the microvesicular hyperplastic polyps, and $60(71 \%)$ of them showed nuclear atypia $\left(P=0.003, \chi^{2}\right.$ test). Nevertheless, 15 (43\%) of the 35 VE1 microvesicular hyperplastic polyps also displayed nuclear atypia. No nuclear atypia was present in goblet-cell-rich hyperplastic polyps (Table 4).

\section{Sequencing for $B R A F$ and $K R A S$ Mutations}

Sequencing for BRAF was performed in 30 cases, comprising 10 VE1 - (8 microvesicular hyperplastic polyps, 1 traditional serrated adenoma, 1 conventional adenoma) and $20 \mathrm{VE} 1+$ samples (6 sessile serrated adenomas/polyps, 9 microvesicular hyperplastic polyps, 5 traditional serrated adenoma), of which 1 microvesicular hyperplastic polyp showed weak and heterogeneous immunostaining.

All VE1 - samples showed no BRAF exon 15 mutation. Of the 20 VE1 + samples, 2 microvesicular hyperplastic polyps with a low number of positive crypts were $B R A F$ wild-type at direct sequencing, but after BRAF enrichment using a V600E kit (Qiagen GmbH, Düsseldorf, Germany), V600E mutations were evident. The remaining 17 VE1 + samples (among them also the one with weak immunostaining) showed V600E mutations at direct sequencing. Interestingly, one VE1 weakly positive microvesicular hyperplastic polyp showed BRAF V600Q (c.1799_1800delinsAA) at sequencing.

Sequencing for KRAS was performed in 41 samples, of which 33 were VE1 - (17 microvesicular hyperplastic polyp, 11 goblet-cell-rich hyperplastic polyp, 1 traditional serrated adenoma, 4 conventional adenoma) and 8 VE1 + (4 sessile serrated adenoma/polyp, 3 microvesicular hyperplastic polyp, 1 traditional serrated adenoma). In VE1 - cases, 2 microvesicular hyperplastic polyp and 1 conventional adenoma showed KRAS exon 2 mutations, whereas in the VE1 + samples, no KRAS exon 2 mutations were found. 

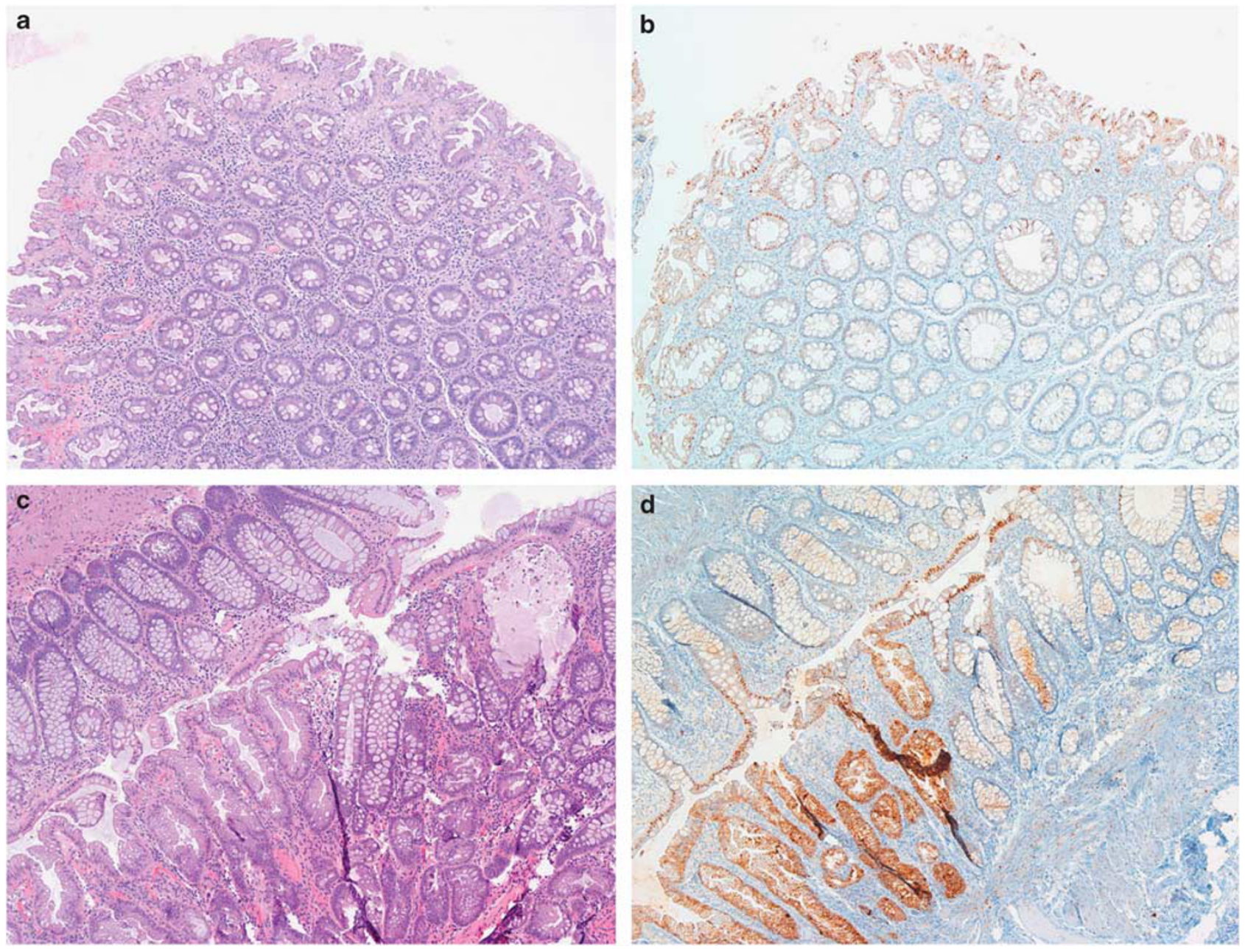

Figure 2 Microvesicular hyperplastic polyp with mild nuclear atypia: (a) hematoxylin and eosin (H\&E), $\times 100$; (b) Immunostain VE1 $(-), \times 100$; (c) H\&E, $\times 100$; and (d) Immunostain VE1 $(+), \times 100$.

\section{Discussion}

The development of a BRAF V600E mutation-specific antibody has enabled us to investigate for the first time the distribution of BRAF V600E protein in routine formalin-fixed, paraffin-embedded specimens of serrated lesions of the colon. This approach allows a correlation of histomorphology with molecular pathological changes at the cellular level and an immunohistochemical subclassification of serrated lesions according to their BRAF V600E mutation status.

In this study, we propose a novel and refined classification for serrated lesions of the colon integrating histomorphology with immunohistochemical detection of BRAF V600E mutation. This technique provides an objective and reproducible aid to identify serrated lesions with the oncogenic BRAF V600E mutation.

BRAF V600E mutation is an early event in the serrated neoplasia pathway. ${ }^{12}$ It has been anticipated that microvesicular hyperplastic polyps are the precursor lesions for the serrated pathway and might progress into sessile serrated adenoma/polyp and to colorectal cancer. ${ }^{9,15,16}$ Our findings, on one hand identifying $(71 \%)$ microvesicular hyperplastic polyps with the oncogenic BRAF V600E mutation and on the other hand the presence of VE1 + areas revealing the morphology of microvesicular hyperplastic polyps in 95\% of sessile serrated adenomas/ polyps, would favor this theory. The observed abrupt transition in all VE1 + serrated lesions between areas with and without gene mutation also indicates a clonal evolution of the BRAF V600E mutation.

Generally, colorectal cancer is believed to arise on the base of distinct precursor lesions and is associated with different molecular pathways, that is, mutation in the KRAS gene (mainly in exon 2) or in the BRAF gene (in the vast majority of cases the V600E mutation in exon 15). ${ }^{14,29-31}$ In our study, BRAF V600E mutation neither could be detected in goblet-cell-rich hyperplastic polyps nor in conventional adenomas. The latter fact provides evidence that BRAF V600E mutation probably does not have a major role in the conventional adenomacarcinoma sequence, but in a subset of microsatellite-stable colorectal cancer, it has been shown that 

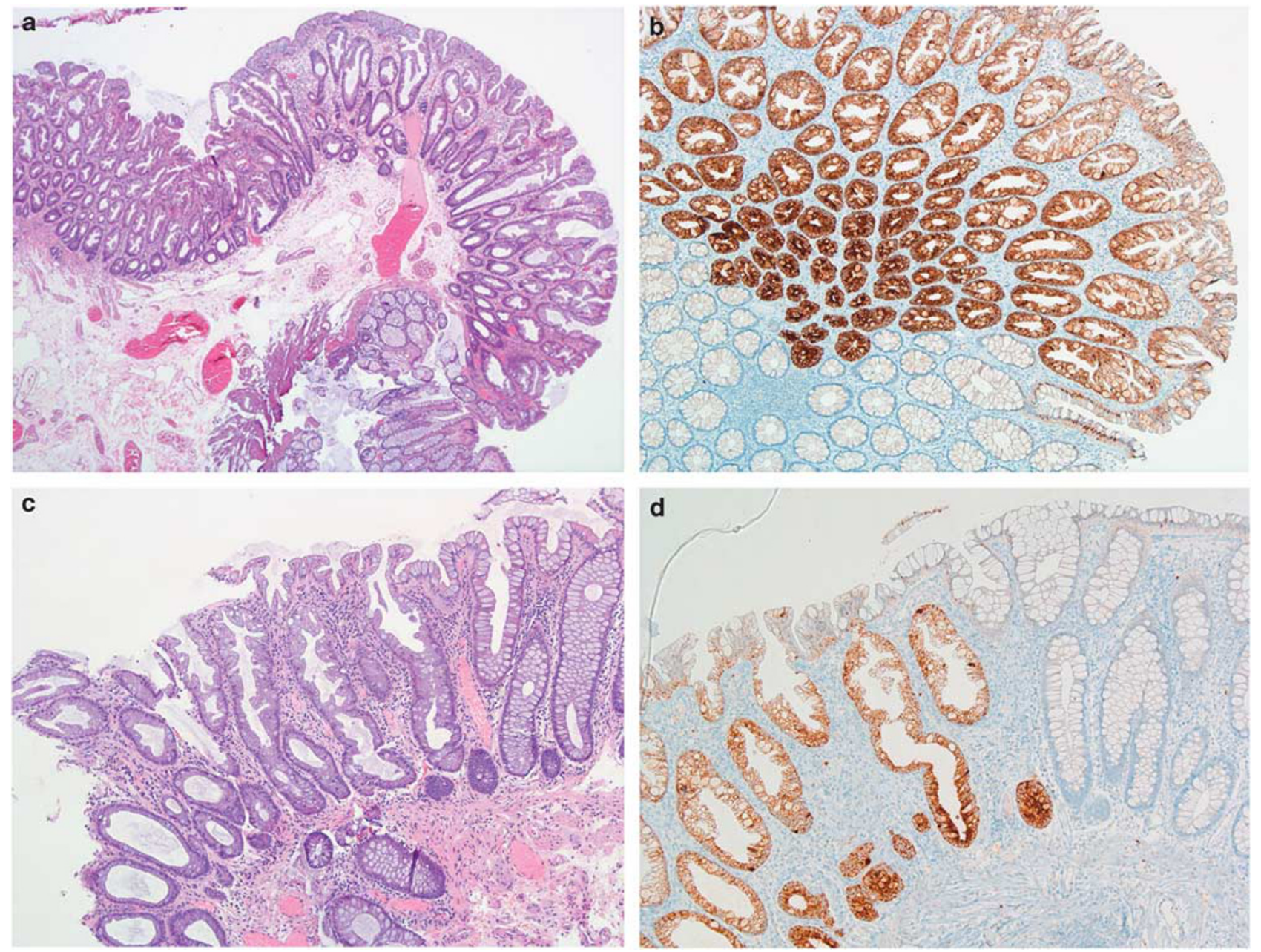

Figure 3 Sessile serrated adenoma/polyp with features of microvesicular hyperplastic polyp: (a) hematoxylin and eosin (H\&E), $\times 40$; (b) immunostain VE1, $\times 100$. Sessile serrated adenoma/polyp without cytologic dysplasia: (c) H\&E, $\times 200 ;($ d) immunostain VE1, $\times 200$.

BRAF V600E mutation does have a relevant role, is prognostically significant and correlates with poorer survival. ${ }^{32,33}$

Although consensus exists that sessile serrated adenomas are precursors of BRAF V600E-driven serrated carcinoma pathway, the contribution of hyperplastic polyps is not unambiguously solved. 9,13,15 These lesions are frequently found and ablated at routine colonoscopy. Their neoplastic nature remains an issue of debate and many attempts have been made to find histomorphologic ${ }^{17}$ and immunomorphologic ${ }^{34}$ criteria to identify among them the neoplastic lesions, in contrast to true hyperplastic lesions. In addition, the histological diagnosis of serrated lesions often poses a difficult task to routine pathologists: biopsy specimens are often small and impossible to orientate. However, proper specimen orientation is crucially important for the differential diagnosis microvesicular hyperplastic polyp vs sessile serrated adenoma/polyp, which is based on architectural changes including the crypt bases. In contrast to conventional adenomas, cytological signs of dysplasia are missing in most cases of sessile serrated adenomas/polyps. Therefore, high interand even intraobserver variability among pathologists is evident in the diagnosis of sessile serrated adenomas/polyps, ${ }^{19-22}$ despite the formally clear morphologic criteria. ${ }^{13,17}$ The need for a potential marker to identify microvesicular hyperplastic polyps with risk for progression in the serrated neoplasia pathway is also highlighted by our findings. Even though BRAF V600E mutation correlated with nuclear atypia and nuclear stratification, these morphologic changes were also present in $43 \%$ of these cases in the absence of BRAF V600E mutation. These findings suggest that morphology by itself is not a reliable criterion for evaluating the presence of underlying molecular changes with regard to BRAF V600E mutation.

Further problems appear by the fact, which was also evident in our study, that serrated lesions with the morphology of microvesicular hyperplastic polyps in one part of the lesion and with the morphology of sessile serrated adenomas/polyps in the other part do exist. ${ }^{13}$ On one hand, this has been 

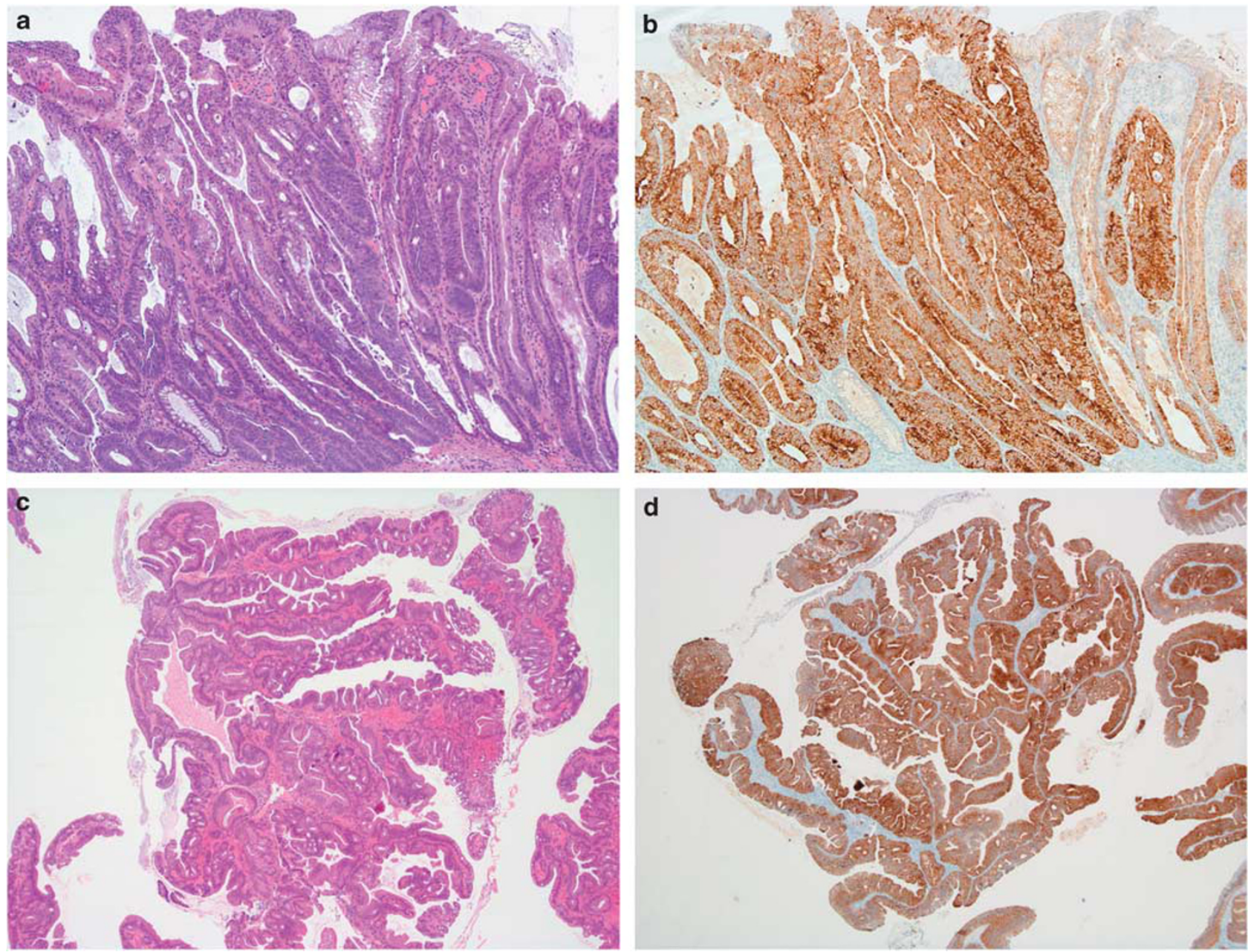

Figure 4 Sessile serrated adenoma/polyp with cytologic dysplasia: (a) hematoxylin and eosin (H\&E), $\times 100$; (b) immunostain VE1, $\times$ 100. Traditional serrated adenoma: (c) H\&E, $\times 100$; (d) immunostain VE1, $\times 100$.

interpreted as evidence that sessile serrated adenomas/polyps evolve from microvesicular hyperplastic polyps or on the other hand that microvesicular hyperplastic polyp features could belong to the morphologic spectrum of sessile serrated adenomas/polyps. ${ }^{13}$ This implicates that there are borderline lesions ${ }^{35}$ and therefore no clear line can be drawn between the morphology of microvesicular hyperplastic polyps and sessile serrated adenomas/polyps. It is likely that the progression between microvesicular hyperplastic polyp and sessile serrated adenoma/polyp is a continuum with BRAF V600E mutation as an early genetic event in the serrated neoplasia pathway. For this reason, it might generally be anticipated that microvesicular hyperplastic polyps with $B R A F$ V600E mutation are not innocent bystanders, but indeed precursor lesions of sessile serrated adenomas/polyps finding their end point in $B R A F$ $V 600 E$-mutated colorectal cancer. It might also be anticipated that serrated lesions including microvesicular hyperplastic polyps with an already existing BRAF V600E mutation have a conside- rable higher risk of progression to malignancy. Further clinical studies of patients with VE1+ microvesicular hyperplastic polyps with appropriate follow-up data could possibly answer this question.

As BRAF V600E is also found frequently in naevi, ${ }^{36-38}$ this finding indicates that non-malignant lesions with this mutation require a 'second hit' to become frankly malignant. This happens obviously only in a relatively small proportion of cases. The same scenario might be true in the development of colorectal cancer as well. In our study, BRAF V600E mutation was encountered in a large percentage (62\%) of hyperplastic polyps almost equally frequent on both sides of the colon, whereas only $5-20 \%$ of colorectal cancers show BRAF V600E mutation and are located more frequently in the right colon. ${ }^{8,14,29,31}$ Therefore, the sole location of a lesion does not seem to be indicative of the underlying molecular pathway. However, the exact mechanisms and host-related factors contributing to further progressions should be addressed in subsequent functional and clinical studies. 
In our opinion, this approach, based on immunohistochemical determination of BRAF V600E mutation, is a feasible technique in routine biopsy diagnostics, allowing unified, objective and reproducible classification of serrated lesions. This would overcome the problems of high interobserver variability using the morphological criteria. Accordingly, microvesicular hyperplastic polyps should be subclassified with regard to BRAF V600E mutation status to identify those with BRAF V600E mutation as possible precursor lesions of the serrated neoplasia pathway. Through this approach, a more elaborate classification based on the interplay between histology and molecular pathology could offer patients an improved colorectal cancer prevention strategy.

\section{Disclosure/conflict of interest}

David Capper and Andreas von Deimling declare shared investorship of VE1 antibody and have applied for a patent on its diagnostic use.

\section{References}

1 Singh H, Turner D, Xue L, et al. Risk of developing colorectal cancer following a negative colonoscopy examination: evidence for a 10-year interval between colonoscopies. JAMA 2006;295:2366-2373.

2 Lakoff J, Paszat LF, Saskin R, et al. Risk of developing proximal versus distal colorectal cancer after a negative colonoscopy: a population-based study. Clin Gastroenterol Hepatol 2008;6:1117-1121; quiz 064.

3 Jass JR. Serrated adenoma of the colorectum and the DNA-methylator phenotype. Nat Clin Pract Oncol 2005;2:398-405.

4 Brenner H, Hoffmeister M, Arndt V, et al. Protection from right- and left-sided colorectal neoplasms after colonoscopy: population-based study. J Natl Cancer Inst 2010;102:89-95.

5 Baxter NN, Goldwasser MA, Paszat LF, et al. Association of colonoscopy and death from colorectal cancer. Ann Intern Med 2009;150:1-8.

6 Vogelstein B, Fearon ER, Hamilton SR, et al. Genetic alterations during colorectal-tumor development. N Engl J Med 1988;319:525-532.

7 Huang CS, O’Brien MJ, Yang S, et al. Hyperplastic polyps, serrated adenomas, and the serrated polyp neoplasia pathway. Am J Gastroenterol 2004;99: 2242-2255.

8 Huang CS, Farraye FA, Yang S, et al. The clinical significance of serrated polyps. Am J Gastroenterol 2010;106:229-240; quiz 41.

9 O’Brien MJ, Yang S, Mack C, et al. Comparison of microsatellite instability, $\mathrm{CpG}$ island methylation phenotype, BRAF and KRAS status in serrated polyps and traditional adenomas indicates separate pathways to distinct colorectal carcinoma end points. Am J Surg Pathol 2006;30:1491-1501.

10 Jass JR, Whitehall VL, Young J, et al. Emerging concepts in colorectal neoplasia. Gastroenterology 2002;123:862-876.
11 Hawkins NJ, Bariol C, Ward RL. The serrated neoplasia pathway. Pathology 2002;34:548-555.

12 Kambara T, Simms LA, Whitehall VL, et al. BRAF mutation is associated with DNA methylation in serrated polyps and cancers of the colorectum. Gut 2004;53:1137-1144.

13 Rex DK, Ahnen DJ, Baron JA, et al. Serrated lesions of the colorectum: review and recommendations from an expert panel. Am J Gastroenterol 2012;107:1315-1329.

14 Davies H, Bignell GR, Cox C, et al. Mutations of the BRAF gene in human cancer. Nature 2002;417: 949-954.

15 O‘Brien MJ. Hyperplastic and serrated polyps of the colorectum. Gastroenterol Clin N Am 2007;36:947-968.

$16 \mathrm{Kim} \mathrm{KM}$, Lee EJ, Ha S, et al. Molecular features of colorectal hyperplastic polyps and sessile serrated adenoma/polyps from Korea. Am J Surg Pathol 2011;35:1274-1286.

17 Torlakovic E, Skovlund E, Snover DC, et al. Morphologic reappraisal of serrated colorectal polyps. Am J Surg Pathol 2003;27:65-81.

18 Snover DC, Ahnen DJ, Burt RW, et al. Serrated polyps of the colon and rectum and serrated polyposis, In: Bosman FT, Carneiro F, Hruban RH, Theise ND (eds) WHO Classification of Tumors of the Digestive System, 4th edn. International Agency for Research on Cancer: Lyon, France; 2010, pp 160-165.

19 Khalid O, Radaideh S, Cummings OW, et al. Reinterpretation of histology of proximal colon polyps called hyperplastic in 2001. World J Gastroenterol 2009;15: 3767-3770.

20 Glatz K, Pritt B, Glatz D, et al. A multinational, internet-based assessment of observer variability in the diagnosis of serrated colorectal polyps. Am J Clin Pathol 2007;127:938-945.

21 Sandmeier D, Seelentag W, Bouzourene H. Serrated polyps of the colorectum: is sessile serrated adenoma distinguishable from hyperplastic polyp in a daily practice? Virchows Arch 2007;450:613-618.

22 Mohammadi M, Garbyal RS, Kristensen $\mathrm{MH}$, et al. Sessile serrated lesion and its borderline variantvariables with impact on recorded data. Pathol Res Pract 2011;207:410-416.

23 Jass JR. Classification of colorectal cancer based on correlation of clinical, morphological and molecular features. Histopathology 2007;50:113-130.

24 Koperek O, Kornauth C, Capper D, et al. Immunohistochemical detection of the BRAF V600E-mutated protein in papillary thyroid carcinoma. Am J Surg Pathol 2012;36:844-850.

25 Capper D, Berghoff AS, Deimling A, et al. Clinical neuropathology practice news 2-2012: BRAF V600E testing. Clin Neuropathol 2012;31:64-66.

26 Capper D, Berghoff AS, Magerle M, et al. Immunohistochemical testing of BRAF V600E status in 1,120 tumor tissue samples of patients with brain metastases. Acta Neuropathol 2012;123:223-233.

27 Snover DC, Ahnen DJ, Burt RW, et al. Serrated polyps of the colon and rectum and serrated polyposis. In: Bosman FT, Carneiro F, Hruban RH, Theise ND, (eds). World Health Organization Classification of Tumours, 4th edn. International Agency for Research on Cancer: Lyon, France, 2010, pp 160-165.

28 Capper D, Preusser M, Habel A, et al. Assessment of BRAF V600E mutation status by immunohistochemistry with a mutation-specific monoclonal antibody. Acta Neuropathol 2011;122:11-19. 
29 Yuen ST, Davies H, Chan TL, et al. Similarity of the phenotypic patterns associated with BRAF and KRAS mutations in colorectal neoplasia. Cancer Res 2002; 62:6451-6455.

30 Velho S, Moutinho C, Cirnes L, et al. BRAF, KRAS and PIK3CA mutations in colorectal serrated polyps and cancer: primary or secondary genetic events in colorectal carcinogenesis? BMC Cancer 2008;8:255.

31 Rajagopalan H, Bardelli A, Lengauer C, et al. Tumorigenesis: RAF/RAS oncogenes and mismatch-repair status. Nature 2002;418:934.

32 Samowitz WS, Sweeney C, Herrick J, et al. Poor survival associated with the BRAF V600E mutation in microsatellite-stable colon cancers. Cancer Res 2005;65:6063-6069.

33 Kakar S, Deng G, Sahai V, et al. Clinicopathologic characteristics, CpG island methylator phenotype, and BRAF mutations in microsatellite-stable colorectal cancers without chromosomal instability. Arch Pathol Lab Med 2008;132:958-964.
34 Owens SR, Chiosea SI, Kuan SF. Selective expression of gastric mucin MUC6 in colonic sessile serrated adenoma but not in hyperplastic polyp aids in morphological diagnosis of serrated polyps. Mod Pathol 2008;21:660-669.

35 Mohammadi M, Kristensen $\mathrm{MH}$, Nielsen $\mathrm{HJ}$, et al. Qualities of sessile serrated adenoma/polyp/lesion and its borderline variant in the context of synchronous colorectal carcinoma. J Clin Pathol 2012;65: 924-927.

36 Pollock PM, Harper UL, Hansen KS, et al. High frequency of BRAF mutations in nevi. Nat Genet 2003;33:19-20.

37 Uribe P, Wistuba II, Gonzalez S. BRAF mutation: a frequent event in benign, atypical, and malignant melanocytic lesions of the skin. Am J Dermatopathol 2003;25:365-370.

38 Yazdi AS, Palmedo G, Flaig MJ, et al. Mutations of the BRAF gene in benign and malignant melanocytic lesions. J Invest Dermatol 2003;121:1160-1162. 